\title{
Estudiantes de la licenciatura en enfermería frente a la investigación
}

Corvera-Martínez J.,, Durán-Avelar A*,, Hernández- García JC*., Pérez-Rodríguez J," Alejo- Lozada JG**, Arellano-Pérez $G^{* *}$.

\footnotetext{
-

- Resumen

- Con el objeto de conocer el interés que por la inves-

- tigación, tienen los estudiantes de la licenciatura de

- enfermería de la Universidad Autónoma de Aguasca-

- lientes, se realizó un estudio observacional descriptivo

- en dos fases, mediante la aplicación de una encuesta

- en la que se estudiaron las variables: género, edad,

- interés por la investigación, conocimiento del objetivo

- de la carrera, conocimiento de que es factible realizar

- el servicio social en investigación y que opinión tienen

- sobre la investigación en la universidad. Resultados.

- Encontramos que el 78\% de los alumnos conocen el

- objetivo de la licenciatura, que el 58\% (140) de los

- alumnos encuestados, refirieron tener interés por la
}

investigación y de ellos el $91.5 \%$ quieren realizar el servicio social en investigación, el 58\% opinan que el nivel de la investigación en la licenciatura de enfermería es medio y $24 \%$ considera que esta atrasado. Discusión. Nosotros encontramos que el interés que tienen los estudiantes de la licenciatura de enfermería de la Universidad Autónoma de Aguascalientes es elevado por lo que es necesario generar estrategias que permitan aprovechar tal interés para iniciar y desarrollar proyectos de investigación trascendentes para los estudiantes, la universidad y la sociedad de nuestro estado. LUXMÉDICA

Palabras clave: investigación en enfermería, estudiantes.

* Estudiantes de la Licenciatura de Enfermería

** Profesores investigadores del depto. de Salud Pública del Centro de Ciencias de la Salud UAA.

Fecha de recibido: 14-junio-2010 Fecha de aceptado: 12-agosto-2010

Correspondencia: Arellano-Pérez G. Doctor en Ciencias. depto. de Salud Pública del Centro de Ciencias de la Salud UAA. Av. Universidad \#940 Ciudad Uniiversitaria cp 20100 Ags. Ags. Tel. 910-84-34. Correo electrónico: Igarella@correo.uaa.mx 


\section{Introducción}

La enfermería ha experimentado un importante desarrollo en los últimos años en Latinoamérica, proceso que se expresa en el aumento progresivo de escuelas de enfermería, la diversidad de programas de posgrado o especialidad, la generación de sociedades, y una multiplicidad de congresos de divulgación disciplinaria ${ }^{1}$. Esto ha generado un incremento en la posibilidad de realizar trabajos de investigación por parte de los profesionales de la enfermería. En México, la mayor parte del personal de enfermería desarrolla trabajos de investigación, por lo general, como un medio para obtener algún grado académico ${ }^{2}$. Sin embargo, aunque el profesional de enfermería ha participado desde hace muchos años en la investigación, invitados generalmente por médicos, éstos han sido ocupados como encuestadores o recolectores de datos clínicos (presión arterial, talla, peso, glicemia) y sin tener un reconocimiento por su participación y menos en la publicación de los artículos ${ }^{3}$. También es evidente que el desarrollo de la investigación realizada por enfermeras ha sido muy lento, en los años setenta se destacaba que sólo el $0.1 \%$ de las enfermeras realizaba labores de investigación ${ }^{4}$, lo cual puede estar influido por que sólo una minoría de los profesionales de la enfermería tenía el nivel de licenciatura, proceso que en la actualidad tiende a revertirse con el incremento de licenciados en enfermería ejerciendo la profesión, con lo que es factible se incremente el número de publicaciones por parte de los profesionales de la enfermería. En el presente trabajo quisimos conocer el interés que por la investigación, tienen los estudiantes de la licenciatura de enfermería de la Universidad Autónoma de Aguascalientes..

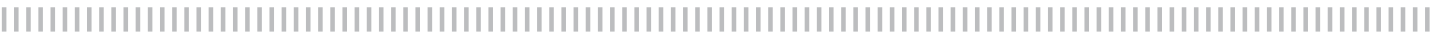

\section{Material y métodos}

Se realizó un estudio descriptivo, observacional, en dos fases mediante la aplicación de una encuesta previamente validada mediante prueba piloto. En una primera fase se encuestaron a los 240 alumnos de la licenciatura de enfermería inscritos en el semestre enero-junio de 2010, en la Universidad Autónoma de Aguascalientes para conocer la proporción de los que presentaban algún interés por la investigación, y posteriormente a los mismos se les aplicó una segunda encuesta en la cual se buscaron las siguientes variables: género, edad, semestre que cursan, interés por la investigación, conocimiento del objetivo de la carrera, conocimiento del porcentaje de la carga académica del plan de estudios de enfermería dedicado a la investigación, conocimiento del perfil de egreso de la carrera de enfermería, conocimiento de que es factible realizar el servicio social en investigación, conocimiento de que durante la carrera tiene que desarrollar trabajos de investigación, qué opinión tienen sobre la investigación en la universidad, cuál es su opinión sobre el nivel de preparación que tienen los profesores de la carrera en el área de investigación y en qué área de las que abarca el desarrollo del profesional de enfermería quisiera desarrollarse.

Para el análisis estadístico se obtuvieron únicamente porcentajes. Se excluyeron 15 encuestas en la segunda fase debido a su mal llenado por parte de los alumnos. 


\section{Resultados}

En la primera fase se encontró que el $58 \%$ (140/240) tiene gusto por la investigación y al $38 \%$ le agradan más las áreas de docencia, administración y asistencial. Sin embargo el $64.5 \%$ de ellos sabe que tiene la obligación de aportar productos de investigación.

En base a los datos obtenidos durante la segunda fase del estudio se obtuvieron los siguientes resultados: el interés de los alumnos por realizar servicio social en áreas de investigación es del 91.5\% (128/140), el $58 \%$ piensa que el nivel de la investigación que se realiza en la carrera de licenciado en enfermería es medio y el $24 \%$ contestó que se encuentra atrasado.

El 78\% de los encuestados conoce el objetivo de la licenciatura, teniendo en cuenta que este objetivo menciona que una de las tareas para el desarrollo, del profesional de la enfermería es la investigación.

En relación a la opinión que ellos expresan sobre ¿cuál área debe mejorarse en la carrera para avanzar en la investigación?, el $43 \%$ menciona que se deben realizar mejoras en la enseñanza, el $23 \%$ opina que se debe mejorar la docencia y el $26 \%$ considera que se debe mejorar el perfil del estudiante.

Respecto a la opinión sobre el nivel académico de los docentes de la carrera en el área de investigación; el $47 \%$ consideró que tienen buen nivel académico, 38\% tienen un nivel regular, $3 \%$ malo y sólo el $12 \%$ considera que sus maestros tiene un muy buen nivel académico.

Respecto al interés por la investigación según el género del estudiante se encontraron frecuencias similares, ya que el $42.9 \%$ de los hombres y el $57.1 \%$ de las mujeres se interesan por la investigación, aunque la diferencia entre ambos no es significativa.

Así mismo se encontró que el $46.3 \%$ de los alumnos desconoce si existe apoyo de la universidad para que realicen investigación.

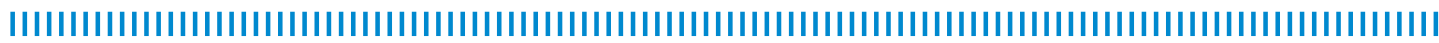

\section{Discusión}

De los datos obtenidos podemos observar que de los alumnos de la carrera de enfermería de nuestra universidad un poco más de la mitad muestra interés por la investigación, y un elevado porcentaje de ellos considera como relevante la posibilidad de realizar el servicio social en el contexto de un trabajo de investigación, esto indudablemente ofrece una importante oportunidad para el desarrollo de trabajos de investigación en la carrera y lo cual debe orientar a que la universidad aprovecha tal interés para desencadenar líneas de investigación con alumnos como autores o colaboradores de trabajos de investigación que en la medida que se desarrollen pueden impactar en la salud de nuestra sociedad y de la comunidad estudiantil.
Como lo refiere Benavides Torres y colaboradores ${ }^{5}$, la profesión de enfermería no llegará a ser reconocida como tal mientras no se explote la investigación en la etapa de formación de sus estudiantes.

Es indispensable y obligatorio que el profesional de la enfermería, realice aportes para cambiar la perspectiva social y de los mismos integrantes del equipo de salud, está en las instituciones y en la mentalidad de los alumnos despertar por completo el interés por la investigación y no solo por el área asistencial.

Consideramos que para aprovechar el interés que los alumnos de la licenciatura en enfermería para integrarse en proyectos de investigación en el Centro de Ciencias de la Salud se debe:

1. Incrementar la carga porcentual de las 
materias relacionadas con la investigación en el plan de estudios de la licenciatura de enfermería cuando se realice la próxima revisión del mismo.

2. Realizar una difusión efectiva de los programas, congresos y becas relacionadas con la investigación en el área de enfermería.

3. Crear espacios dedicados al desarrollo de proyectos de enfermería.

\section{Bibliografía}

1 Alarcón AM, Astudillo PD. La investigación en enfermería en revistas latinoamericanas. Ciencia y Enfermería 2007; 13 (2):25-31.

2 Crispin AL. Una reflexión sobre el desarrollo de la investigación en enfermería. Desarrollo científico de la enfermería. 2009; 17 (3):1-2.

3 Salazar-Gómez T, Landeros-Olvera EA, Salas-Martínez $\mathrm{O}$, Enríquez-González Q. Financiamiento de la
4. Favorecer que los docentes de la licenciatura de enfermería fomenten la participación de los alumnos en proyectos de investigación.

5. Incrementar el número de docentes de la carrera de enfermería con perfil PROMEP y que pertenecen al sistema nacional de investigadores.

investigación en enfermería: beneficios y barreras. Rev Enferm Inst Mex Seguro Soc. 2008;16(1):51 55

4 XIII Coloquio Difusión de investigación en enfermería. Facultad de Enfermería y Obstetricia de Celaya de la Universidad de Guanajuato. 2005.

5 Benavides Torres RA. La investigación en la enfermería mexicana. Rev. Enferm Inst Mex Seguro Soc. 2002;10(3):153-158. 